\title{
Recovery of Lithium from Aqueous Solution using Protonated Potassium Polytitanate
}

\author{
Maria Vikulova $^{1}$, Alexander Gorokhovsky ${ }^{2}$, Elena Tretyachenko ${ }^{3}$, Tatiana Blagova ${ }^{4}$ \\ Department of Chemistry and Chemical Technology of Materials, \\ Yuri Gagarin State Technical University of Saratov \\ 410054 Russia, Saratov, 77 Politechnicheskayastreet \\ 1'vikulovama@yandex.ru \\ 2algo54@mail.ru \\ 3trev07@rambler.ru \\ 4blagova-ta@yandex.ru
}

\begin{abstract}
Potassiumpolytitanate(PPT) with different degree of protonation is proposed as a new kind of inorganic adsorbent for lithium ions recovery from natural aqueous solutions. The phase and chemical composition, as well as the particle size distribution of the sorbents are studied using XRD,XRFanalysis, and laser diffraction. The efficiency of lithium ions recovery and the adsorption process kineticsfor are investigatedusing standard solutions of lithium chloride. The sorption capacity and kinetic characterisitcsof the studied materials are estimated using pseudo-first and pseudo-second-order models.
\end{abstract}

Keyword - Potassium Polytitanate, Protonation, Lithium, Kinetics, Sorption

\section{INTRODUCTION}

In recent years, a need for lithium and its new natural sources hasbeen sharply increased. This is dueto the fact that lithium is an indispensable element in the production of electrode materials for batteries used in portable electronics, hybrid cars and electric vehicles. On the other hand, lithium is widely used in ceramics, glasses, lubricating compositions, metal alloys, air cleaning and other areas.

According to the experts, land resources represented by ores or brines contain more than 14 million tons of lithium [1]. Although the amount of lithium in these resources is sufficient for the current consuming, it is necessary to prepare new technologies to use alternative sources and meet growing demands expected in the near future. As an example of such sources it is possible to consider sea water containing up to 230 billion tons of lithium, although the concentration of alkali metal in it is rather low (0.1-0.2 mg/l) [2].

It is known that pyrometallurgy and hydrometallurgy processes can be used to extract lithium from primary and secondary resources. Although pyrometallurgical processes have relatively low exploitation cost, they require intensive monetary investments and are accompanied by undesirable environmental pollution. Hydrometallurgical processes, including solvent extraction, adsorption (ion exchange) and precipitation, are promising methods for extracting lithium in the form of pure lithium carbonate $\left(\mathrm{Li}_{2} \mathrm{CO}_{3}\right)$ and lithium hydroxide $(\mathrm{LiOH})$ due to a low energy cost and minor waste production. However, Li (I) exists in the natural aqueous solutions altogether with other metal ions, such as $\mathrm{Na}$ (I), K (I), Ca (II), Mg (II), Fe(III), etc. Therefore, the existing impurities must be removed, otherwise the product will have low purity due to co-precipitation of other metals.

Although the represented in the market commercial reagent allow recovering of Li from acidic aqueous solutions with high separation factor between lithium and other monovalent metal cations, a use of these materials is limited due to their low extraction efficiency. Therefore, for sources in which the concentration of lithium ions is low and concentration of other cations is high, reactions based on adsorption and ion exchange are more effective.

There are a number of inorganic ion-exchange materials with extremely high selectivity only for lithium ions. Potential sorbents for the extraction of lithium from water resources are compounds with a spinel structure of Li-Mn-O composition. Depending the methods of synthesis, the adsorption and ion-exchange capacities for extracting lithium from various water resources vary from 1.5 to $55.0 \mathrm{mg} / \mathrm{g}$ [3-14].

However, nowadays, the titanium oxides are considered as promising sorbents due to their greater stability, in comparison with themanganates, in the technological processes accompanying with desorption of lithium, which, obviously, are applied for the following extraction of $\mathrm{Li}$ and regeneration of the sorbent. At the same time, the structure of the crystal lattice of hydrated titanium oxide (or metatitanic acid) determines its selectivity with respect to lithium ions in a presence of such cations as sodium, potassium, magnesium, and calcium ions, typical inthe saline lakes [15]. 
Protonated forms of potassium polytitanate (PPT) are similar in their composition and structure with metatitanic acid and can become a more attractive alternative due to low cost of synthesis and extremely high sorption capacity. At the same time, the structural characteristics of the protonated PPT can be varied by adjusting the protonation conditions. It is assumed that asubstitution degree of the potassium ions by hydronium ions presented in the protonated form of PPT will affect the amount of lithium ions extracted from aqueous solution, and, as a consequence, influence the composition and structure of lithiated products obtained during lithium ions recovery.

Thus, in this work, it is proposed to use protonated potassium polytitanates as a new inorganic ion exchangers, effective for extracting lithium from aqueous solutions. Amorphous potassium polytitanate (PPT) due to its high imperfection and layered structure is an effective ion exchanger and sorbent for ions of various metals. However, aqueous dispersions of PPT have high $\mathrm{pH}$ value, and in the neutral or acidic media potassium polytitanate particles uncontrollably changes their chemical composition due to replacement of metal ions by protons / hydronium ions. That is why; the goal of this work is to recognize the effect of the protonation degree, controlled by $\mathrm{pH}$ value of preliminary treatment, on efficiency of lithium extraction from aqueous solution of its salt.

\section{EXPERIMENTAL}

Protonated potassium polytitanates were obtained by the treatment of aqueous dispersion of the parent potassium polytitanate (PPT), characterized by strong alkaline reaction $(\mathrm{pH} \sim 10.5)$, with aqueous solution of sulphuric acid, till obtaining a stable $\mathrm{pH}$ value equal to $2 ; 3 ; 4 ; 5 ; 6$; and 7 . The obtained powders were centrifuged, dried at $40{ }^{\circ} \mathrm{C}$ for $4 \mathrm{~h}$ and grinded up. The protonated forms of the produced PPT derivatives were marked as PPTP2, PPTP3, PPTP4, PPTP5, PPTP6, PPTP7, in accordance with $\mathrm{pH}$ value of their aqueous dispersions, and further characterized by XRD (diffractometer ARL X'TRA), laser diffraction (particle size analyzerLaser Analyzerte 22 Micro Tec plus) and X-ray fluorescence analysis (X-ray fluorescence spectrometer SPECTROSCAN MAX-GV).

To study the sorption kinetics and adsorption capacity of the produced PPT modifications, the suspensions of protonated potassium polytitanates $(5 \mathrm{~g} / \mathrm{l})$ was mixed with lithium chloride solutions $([\mathrm{Li}]=10 \mathrm{mmol} / \mathrm{l})$.A change of [Li] was analyzed by potentiometric method every $5,10,20,30,60,120,180$, and 240 minutes using laboratory equipment I-160MP with lithium-selective electrode (ELIS-142Li).

The efficiency of $\mathrm{Li}$ (I) ions extraction by protonated potassium polytitanates was calculated in accordance with (1):

$$
E, \%=\frac{C_{0}-C_{t}}{C_{0}} \cdot 100 \%
$$

where $\mathrm{C}_{0}$ and $\mathrm{C}_{\mathrm{t}}$ are the initial and current concentration of $\mathrm{Li}(\mathrm{I})$ in the solution.

An amount of the adsorbed $\mathrm{Li}$ (in $\mathrm{mmol} / \mathrm{g}$ ) at equilibrium $\left(\mathrm{q}_{\mathrm{e}}\right.$, ) and during the process $\left(\mathrm{q}_{\mathrm{t}}\right)$ were calculated using the formulas (2) and (3):

$$
\begin{aligned}
& q_{e}=\frac{\left(C_{0}-C_{e}\right) \cdot V}{m} \\
& q_{t}=\frac{\left(C_{0}-C_{t}\right) \cdot V}{m}
\end{aligned}
$$

where $\mathrm{C}_{\mathrm{o}}, \mathrm{C}_{\mathrm{t}}$ and $\mathrm{C}_{\mathrm{e}}$ correspond to [Li] in the initial, currentand equilibrium condition, respectively; $\mathrm{V}$ is a volume of the solution (1); $\mathrm{m}$ is a weight of the PPT powder used as the adsorbent (g).

The obtained experimental data were processed using the kinetic equations corresponding topseudo-first- and pseudo-second-order models (equations4 and 5):

$$
\lg \left(q_{e}-q_{t}\right)=\lg q_{e}-\frac{k_{1}}{2.303} t
$$

where $\mathrm{k}_{1}$ is pseudo-first-order rate coefficient, $1 / \mathrm{min}$.

$$
\frac{t}{q_{t}}=\frac{1}{k_{2} q_{e}^{2}}+\frac{t}{q_{e}}
$$

where $\mathrm{k}_{2}$ is pseudo-second-order rate coefficient, $\mathrm{g} / \mathrm{mmol} \cdot \mathrm{min}$. 


\section{RESULTS AND DISCUSSION} $1)$.

In accordance with XRD data, all the protonated potassium polytitanates are X-ray amorphous materials (Fig.

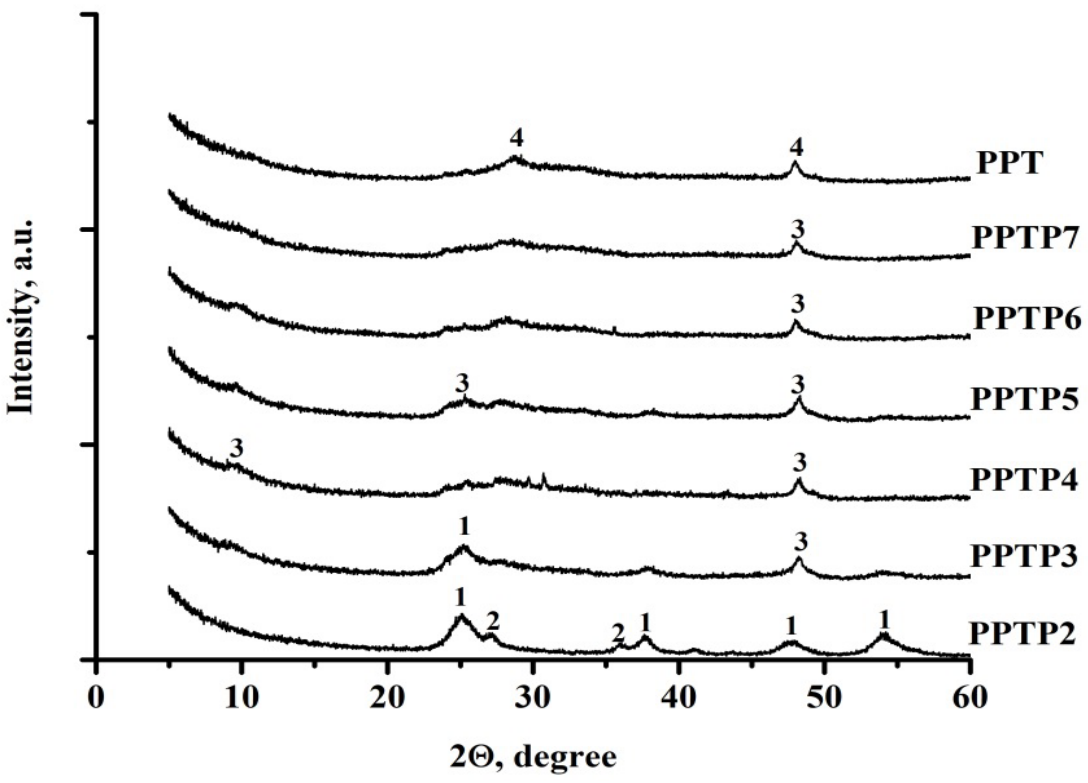

Fig. 1. XRD date of potassium polytitanates: 1 - $\mathrm{TiO}_{2}$ (anatase); $2-\mathrm{TiO}_{2}$ (rutile); $3-\mathrm{K}_{0,48} \mathrm{H}_{0,22} \mathrm{Ti}_{1,825} \mathrm{O}_{4}\left(\mathrm{H}_{2} \mathrm{O}\right)_{0,52} ; 4-\mathrm{K}_{2} \mathrm{Ti}_{2} \mathrm{O}_{5}$

Onlyweak and wide reflections corresponding to $\mathrm{K}_{2} \mathrm{Ti}_{2} \mathrm{O}_{5}$ and crystalline forms of $\mathrm{TiO}_{2}$ (for PPTP3 and PPTP4) could be identified. The appearance of $\mathrm{TiO}_{2}$ crystals at low $\mathrm{pH}$ can be explained by intensive $\mathrm{K}^{+} \leftrightarrow$ $\mathrm{H}_{3} \mathrm{O}^{+}$ion-exchange taking place at these conditions favouring formation of unstable titanium acid, which decomposes producing crystallized titanium dioxide. In the case of samples protonated at higher $\mathrm{pH}$ values, the reflectionscan be considered as corresponding to nanoscale partially protonated forms of the potassium titanate, $\left(\mathrm{K}_{0.48} \mathrm{H}_{0.22} \mathrm{Ti}_{1.825} \mathrm{O}_{4}\left(\mathrm{H}_{2} \mathrm{O}\right)_{0.52}\right)$.

According to the results of elemental analysis (Table I), a regular decrease from $8.3 \mathrm{wt} \%$ (PPTP7) down to $1.1 \mathrm{wt} . \%$ (PPTP2) in the potassium content was noted.

An average particle size of the protonated potassium polytitanates does not exceed $12 \mu \mathrm{m}$, and a minimal value of this one was obtained for PPTP4 (Table I).

TABLE I. The Chemical Composition of Potassium Polytitanates, Protonated at Different pH Values

\begin{tabular}{|c|c|c|c|c|c|c|}
\hline \multirow{2}{*}{$\begin{array}{c}\text { Sample } \\
\text { name }\end{array}$} & \multicolumn{5}{|c|}{ The content of oxides, wt.\% } & \multirow{2}{*}{$\begin{array}{c}\text { Average particle } \\
\text { size, } \boldsymbol{\mu m}\end{array}$} \\
\cline { 2 - 6 } & $\mathbf{T i O}_{\mathbf{2}}$ & $\mathbf{S O}_{\mathbf{3}}$ & $\mathbf{K}_{\mathbf{2}} \mathbf{O}$ & $\mathbf{A l}_{\mathbf{2}} \mathbf{O}_{\mathbf{3}}$ & $\mathbf{S i O}_{\mathbf{2}}$ & 12 \\
\hline PPTP2 & 93,9 & 3,1 & 1,1 & 1,1 & 0,2 & 11 \\
\hline PРTP3 & 95,0 & 1,6 & 2,5 & 0,5 & 0,2 & 7 \\
\hline PРTP4 & 93,7 & 1,8 & 3,5 & 0,7 & 0,2 & 11 \\
\hline PРTP5 & 93,5 & 0,1 & 4,8 & 0,9 & 0,2 & 10 \\
\hline PРTP6 & 90,0 & 0,4 & 8,0 & 0,9 & 0,2 & 8 \\
\hline PРTP7 & 90,2 & 0,1 & 8,3 & 0,7 & 0,2 & 10 \\
\hline
\end{tabular}

The kinetics of Li (I) ions extraction by protonated potassium polytitanatesis shown in Fig.2. 


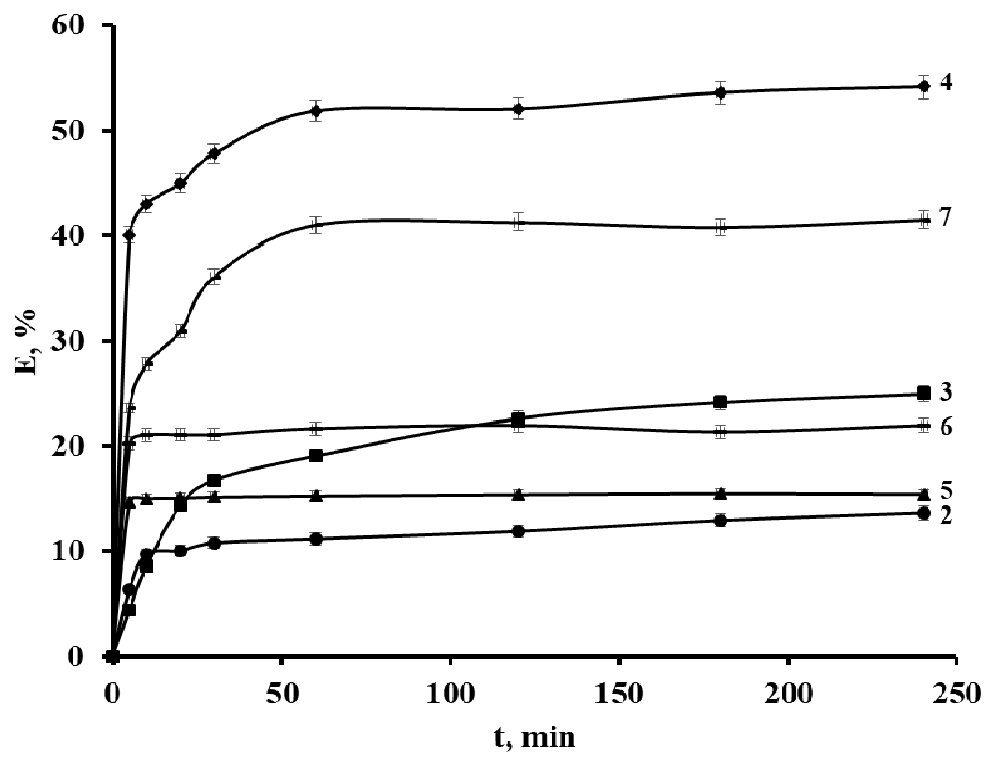

Fig. 2. Time dependency of $\mathrm{Li}$ (I) ions sorption efficiency by different protonated potassium polytitanates

It is observed that an amount of $\mathrm{Li}$ (I) ions removedby all the investigated sorbentsincreases with a time. For the most samples, quasi-equilibrium conditions of the adsorption processes occur within 5-30 minutes (PPTP2, PPTP5, PPTP6). In the case of potassium polytitanates protonated at $\mathrm{pH} \sim 4.0$ and 7.0, the $\mathrm{Li}$ (I) ions residual concentration in the solution practically does not change after 60 minutes. Li (I) ions extraction by PPTP 3 is gradually increases and reaches a value of about $25 \%$. Maximum efficiency of Li (I) ions extraction ( $\sim 54 \%)$ is typical for PPTP4. Presumably this is due to a small average particle size and well developed specific surface area.

The models of chemical kinetics (pseudo-first- and pseudo-second-order) were used to suggest the mechanisms of $\mathrm{Li}$ (I) sorption by different forms of protonated potassium polytitanate powders as well as determine an approximate maximum sorption capacity.

Pseudo-first- and pseudo-second-order kinetics plots for the sorption of Li (I) ions by different PPTPforms are shown in Fig.3 and 4.

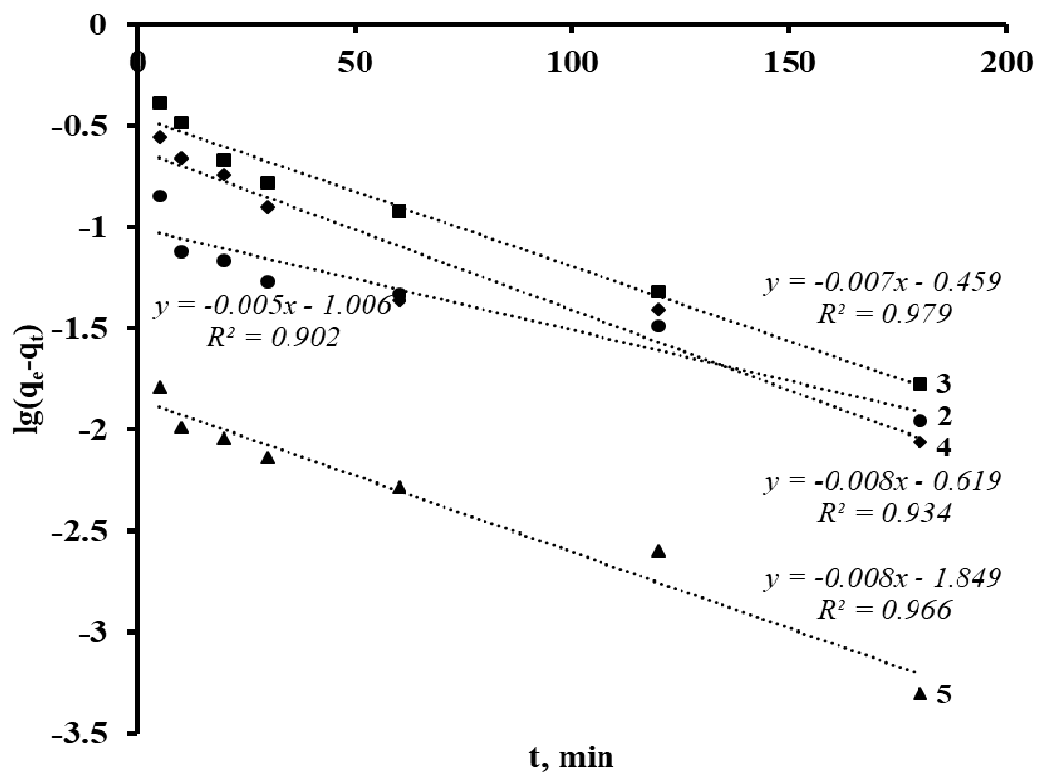

Fig. 3. Pseudo-first-order kinetic plot for sorption of $\mathrm{Li}$ (I) ions by protonated potassium polytitanates 
The experimental sorption data are in a good agreement with the pseudo-first-order model (approximation coefficient $\mathrm{R}^{2}>0.9$ )only in the case of the potassium polytitanates synthesized at $\mathrm{pH}$ varied in the range from 2.0 to 5.0. Consequently, Li (I) ions sorption by protonated potassium polytitanates is preceded by a diffusion stage. In addition, in this case, the process rate is affected only by the amount of one reagent, namely Li (I) ions [16].

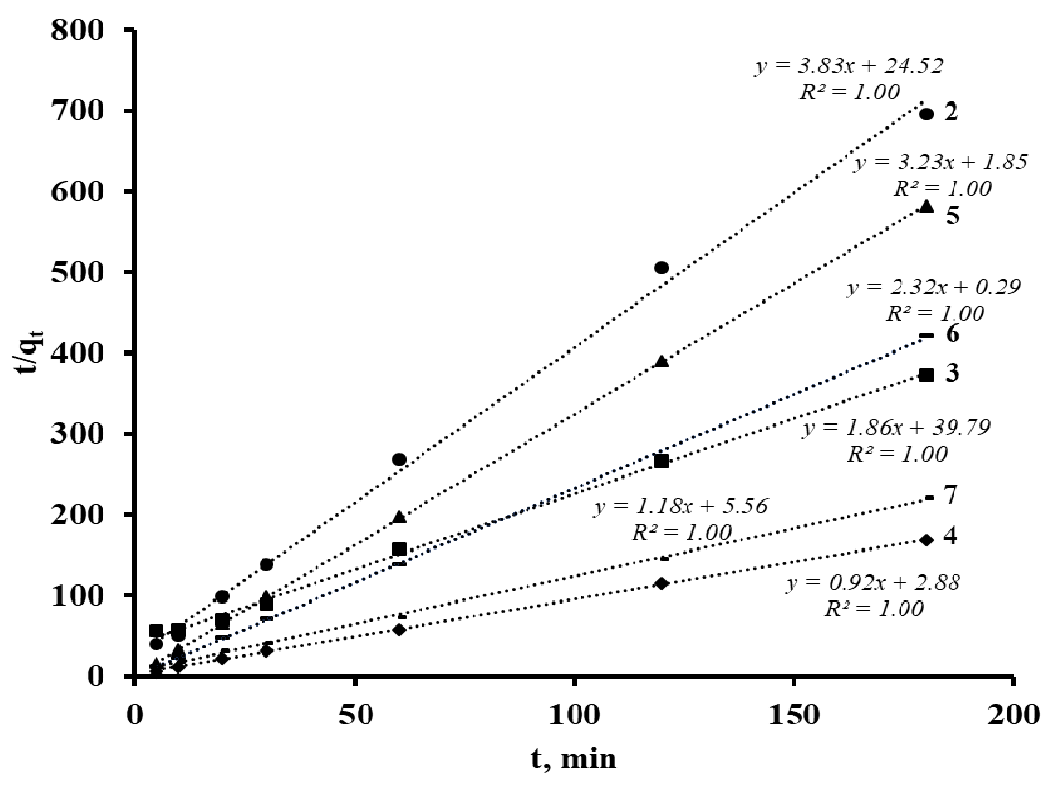

Fig. 4. Pseudo-second-order kinetic plot for sorption of $\mathrm{Li}$ (I) ions by protonated potassium polytitanates

The kinetic plot obtained in the coordinates of pseudo-second-order modelhas linear character (approximation coefficient $\mathrm{R}^{2} \sim 1$ ). According to the pseudo-second-order model, the interaction of protonated potassium polytitanatewith $\mathrm{Li}$ (I) ions, regardless $\mathrm{pH}$ value during the PPTP synthesis,can be considered as a chemical ionexchange reaction [17].

Linear dependencies within the used models allow us to determine the kinetic rate constants graphically. These kinetic parameters as well as the sorption capacity obtained from the first- and second-order kinetic model plots are reported in Table II.

TABLE III. Pseudo-first- and Pseudo-second-order Rate Constants for Li (I) Sorption by PPTP

\begin{tabular}{|c|c|c|c|c|c|c|c|}
\hline \multirow{3}{*}{$\begin{array}{c}\text { Sample } \\
\text { name }\end{array}$} & \multicolumn{6}{|c|}{ Calculated values } & \multirow{3}{*}{$\begin{array}{c}\begin{array}{c}\text { Experimental } \\
\text { values }\end{array} \\
\mathrm{q}_{\mathrm{e}}, \mathrm{mmol} / \mathrm{g} \\
\end{array}$} \\
\hline & \multicolumn{3}{|c|}{ Pseudo-first order } & \multicolumn{3}{|c|}{ Pseudo-second order } & \\
\hline & $\mathbf{R}^{2}$ & $k_{1}, \min ^{-1}$ & $\mathrm{q}_{\mathrm{e}}, \mathrm{mmol} / \mathrm{g}$ & $\mathbf{R}^{2}$ & $\mathrm{k}_{2}, \mathrm{~g} \cdot \mathrm{mmol}^{-1} \cdot \mathrm{min}^{-1}$ & $\mathrm{q}_{\mathrm{e}}, \mathrm{mmol} / \mathrm{g}$ & \\
\hline PРTP2 & 0.902 & 0.012 & 0.099 & \multirow{6}{*}{1.00} & 0.599 & 0.261 & 0.273 \\
\hline PPTP3 & 0.979 & 0.016 & 0.348 & & 0.087 & 0.538 & 0.499 \\
\hline PPTP4 & 0.934 & 0.018 & 0.240 & & 0.294 & 1.087 & 1.083 \\
\hline PPTP5 & 0.966 & 0.018 & 0.014 & & 5.625 & 0.310 & 0.308 \\
\hline РPТР6 & - & - & - & & 18.563 & 0.431 & 0.440 \\
\hline PРTP7 & - & - & - & & 0.251 & 0.847 & 0.830 \\
\hline
\end{tabular}

From Table II data, the correlation coefficients for the pseudo-first-order kinetic model were found to be low compared to the data of pseudo-second-order. Moreover, a large difference in equilibrium sorption capacity $\left(\mathrm{q}_{\mathrm{e}}\right)$ measured directly in the experiments(Fig.2) and calculated on the base of the pseudo-first-order model indicates a poor fit of this model to the experimental data in comparison with the pseudo-second-order one.

Thus, the experimental kinetic data are best fitted to the pseudo-second-order equation and Li (I) sorption by PPTP can be considered as chemisorption, and the chemical reaction is a rate-limiting step.

It should be noted that the maximum sorption rate is obtained for the PPTP6 sample, but the maximum sorption capacity of $1.087 \mathrm{mmol} / \mathrm{g}$ or $46.1 \mathrm{mg} / \mathrm{g}$ is observed at PPTP4. This value of the sorption capacity is comparable to the characteristics of the best sorbents for the extraction of lithium reported in the literature $[3,7$, $10]$. 


\section{Conclusion}

The obtained results confirm that the protonated forms of the amorphous layered potassium polytitanate (PPTP) represent a new effective inorganic sorbent for the extraction of lithium ions from aqueous solutions. The PPTP samples obtained during the treatment of the parent potassium polytitanate $(\mathrm{pH} \sim 10.5)$ in the sulfuric acid aqueous solution at $\mathrm{pH}$ value varied from 2.0 to 7.0 retain an amorphous structure of the initial PPT but are characterized with increased specific surface area. The highest adsorption capacity is recognized for the PPTP powders modified at $\mathrm{pH} \sim 4$. It is assumed that these conditions favor obtaining the PPT powder characterized with optimal combination of the specific area and average size of particles. The kinetics of the Li ions sorption by PPTP is in a good agreement with the pseudo-second-order model. This one indicates that in the adsorption process can be considered as ion-exchange chemical reaction and allows to estimate the equilibrium adsorption capacity of PPTP4 equal to $1.1 \mathrm{mmol} / \mathrm{g}$. The obtained characteristics correspond to the best similar materials mentioned in the literature, have relatively low cost and very high rate of Li extraction. The last is considered as a result of large interlayer distance in the structure of protonated potassium polytitanates.

\section{REFERENCES}

[1] H.Hamzaoui, A.M'nif, H.Hammi, and R.Rokbani, "Contribution to the lithium recovery from brine," Desalination, vol. 158, pp. 221224, Aug. 2003.

[2] G. J. Brunskill, I. Zagorskis, and J. Pfitzner, "Geochemical mass balance for lithium, boron, and strontium in the Gulf of Papua, Papua New Guinea (Project TROPICS)," Geochimica et cosmochimicaacta, vol. 67, pp. 3365-3383, Sep. 2003.

[3] S. Y.Sun,X. Song, Q. H.Zhang,J.Wang, andJ. G. Yu, "Lithium extraction/insertion process on cubic Li-Mn-O precursors with different Li/Mn ratio and morphology," Adsorption, vol. 17, pp. 881-887, Oct. 2011.

[4] R.Chitrakar, Y. Makita, K.Ooi, and A.Sonoda, "Selective uptake of lithium ion from brine by $\mathrm{H}_{1.33} \mathrm{Mn}_{1.67} \mathrm{O}_{4}$ and $\mathrm{H}_{1.6} \mathrm{Mn}_{1.6} \mathrm{O}_{4}$," Chemistry Letters, vol. 41, pp. 1647-1649, 2012.

[5] Q. H. Zhang, S. P. Li, S. Y. Sun, X. S. Yin, and J. G. Yu, " $\mathrm{LiMn}_{2} \mathrm{O}_{4}$ spinel direct synthesis and lithium ion selective adsorption," Chemical Engineering Science, vol. 65, pp. 169-173, Jan. 2010.

[6] Q. H. Zhang, S. Sun, S. Li, H. Jiang, and J. G. Yu, "Adsorption of lithium ions on novel nanocrystal $\mathrm{MnO}_{2}$," Chemical Engineering Science, vol. 62, pp. 4869-4874, Sept.-Oct. 2007.

[7] R.Chitrakar, H.Kanoh, Y.Miyai, and K. Ooi, "Recovery of lithium from seawater using manganese oxide adsorbent $\left(\mathrm{H}_{1.6} \mathrm{Mn}_{1.6} \mathrm{O}_{4}\right)$ derived from $\mathrm{Li}_{1.6} \mathrm{Mn}_{1.6} \mathrm{O}_{4}$, " Industrial \& engineering chemistry research, vol. 40, pp. 2054-2058, Apr. 2001.

[8] L. Liu, H. Zhang, Y. Zhang, D. Cao, and X. Zhao, "Lithium extraction from seawater by manganese oxide ion sieve $\mathrm{MnO}_{2} \cdot 0.5 \mathrm{H}_{2} \mathrm{O}$," Colloids and Surfaces A: Physicochemical and Engineering Aspects, vol. 468, pp. 280-284, Mar. 2015.

[9] X. Shi,D. Zhou,Z. Zhang,L. Yu,H. Xu,B. Chen, andX. Yang,"Synthesis and properties of $\mathrm{Li}_{1.6} \mathrm{Mn}_{1.6} \mathrm{O}_{4}$ and its adsorption application," Hydrometallurgy, vol. 110, pp. 99-106, Dec. 2011.

[10] J. L. Xiao, S. Y. Sun, J. Wang, P. Li, and J. G. Yu, "Synthesis and adsorption properties of $\mathrm{Li}_{1.6} \mathrm{Mn}_{1.6} \mathrm{O}_{4}$ spinel," Industrial \& Engineering Chemistry Research, vol. 52, pp 11967-11973, 2013.

[11] K.Ooi, Y. Makita, A.Sonoda, R.Chitrakar, Y. Tasaki-Handa, and T. Nakazato, "Modelling of column lithium adsorption from pHbuffered brine using surface $\mathrm{Li}^{+} / \mathrm{H}^{+}$ion exchange reaction," Chemical Engineering Journal, vol. 288, pp. 137-145, Mar. 2016.

[12] T.Ryu, Y.Haldorai, A. Rengaraj, J. Shin, H. J. Hong, G. W. Lee, Y.-K. Han, Y. S. Huh, and K. S. Chung, "Recovery of lithium ions from seawater using a continuous flow adsorption column packed with granulated chitosan-lithium manganese oxide," Industrial \& Engineering Chemistry Research, vol. 55, pp. 7218-7225, 2016.

[13] G.Xiao, K. Tong, L. Zhou, J. Xiao S., Sun, P. Li, and J. Yu, "Adsorption and desorption behavior of lithium ion in spherical PVC$\mathrm{MnO}_{2}$ ion sieve," Industrial \& Engineering Chemistry Research, vol. 51, pp. 10921-10929, 2012.

[14] L. W. Ma, B. Z. Chen, Y. Chen, and X. C. Shi, "Preparation, characterization and adsorptive properties of foam-type lithium adsorbent," Microporous and Mesoporous Materials, vol. 142, pp. 147-153, Jun. 2011.

[15] L. Zhang, D. Zhou, G. He, F. Wang, and J. Zhou, "Effect of crystal phases of titanium dioxide on adsorption performance of $\mathrm{H}_{2} \mathrm{TiO}_{3}-$ lithium adsorbent,"Materials Letters, vol. 135, pp. 206-209, Nov. 2014.

[16] S. K. Lagergren, "About the theory of so-called adsorption of soluble substances," Sven. Vetenskapsakad. Handingarl, vol. 24, pp. 1$39,1989$.

[17] Y. S. Ho, J. C. Y. Ng,andG. McKay, "Kinetics of pollutant sorption by biosorbents." Separation and purification methods, vol. 29, pp. $189-232,2000$

\section{AUTHOR PROFILE}

Maria Vikulova, PhD, Assisting Professor,Department of Chemistry and Chemical Technology of Materials, Yuri Gagarin State Technical University of Saratov, Russia.

Alexander Gorokhovsky, D.Sc. (physical chemistry), Director, Institute of Applied Physics, Yuri Gagarin State Technical University of Saratov, Russia.

Elena Tretyachenko, PhD, Associated Professor, Department of Chemistry and Chemical Technology of Materials, Yuri Gagarin State Technical University of Saratov, Russia.

Tatiana Blagova, 4th year student, Department of Chemistry and Chemical Technology of Materials, Yuri Gagarin State Technical University of Saratov, Russia. 